Case Report

\title{
PR3ANCA Related Cerebral Vasculitis in Ulcerative Colitis Presenting with Orbital Involvement: A Case Report with Review of Literature
}

\author{
Athira Unnikrishnan, ${ }^{1}$ Shila Azodi, ${ }^{2}$ Nadeem Ansari, ${ }^{1}$ Megan Brown, ${ }^{3}$ \\ Joshua Kamnetz, ${ }^{4}$ and Robert C. Uchiyama ${ }^{1}$ \\ ${ }^{1}$ Department of Internal Medicine, University of Texas Southwestern Medical School Residency Programs at Austin, \\ University Medical Center Brackenridge, Austin, TX 78701, USA \\ ${ }^{2}$ Department of Neurology, University of Texas Southwestern Medical School Residency Programs at Austin, \\ University Medical Center Brackenridge, Austin, TX 78701, USA \\ ${ }^{3}$ Transitional Program, University of Texas Southwestern Medical School Residency Programs at Austin, \\ University Medical Center Brackenridge, Austin, TX 78701, USA \\ ${ }^{4}$ School of Medicine, University of Texas Medical Branch, Galveston, TX 77555, USA
}

Correspondence should be addressed to Athira Unnikrishnan; aunnikrishnan17@gmail.com

Received 21 April 2014; Accepted 5 June 2014; Published 2 July 2014

Academic Editor: Tsai-Ching Hsu

Copyright ( 2014 Athira Unnikrishnan et al. This is an open access article distributed under the Creative Commons Attribution License, which permits unrestricted use, distribution, and reproduction in any medium, provided the original work is properly cited.

PR3 ANCA is a classic marker of granulomatosis with polyangiitis (GPA). There have been several recent reports of increased prevalence of PR3ANCA in ulcerative colitis (UC) patients, the clinical implication of which is not well defined. We are reporting a case of 27-year-old Caucasian male with 14-year history of UC presenting with unilateral proptosis, conjunctival congestion, and chemosis who developed acute hemiparesis within three days of hospital admission, followed by rapid neurological deterioration correlating with brain imaging findings. Serologically he had atypical PANCA with high PR3 antibody titer with a negative infectious workup. His cerebral angiogram was normal but the brain biopsy showed necrotizing vasculitis. He was diagnosed with PR3 ANCA mediated cerebral and orbital vasculitis associated with UC. Treatment was initiated with high dose steroids, plasmapheresis, and cyclophosphamide. He improved significantly with residual left hemiparesis.

\section{Introduction}

PR3 ANCA is a classic marker of granulomatosis with polyangiitis (GPA). There have been several recent reports of increased prevalence of PR3ANCA in ulcerative colitis patients, the clinical implication of which is not well defined [1]. In small cohort studies PR3 ANCA has been associated with extensive colitis and shorter disease duration without any gender predilection [2]. However, the incidence of small vessel vasculitis in PR3 ANCA positive UC patient cohort is not known. We are reporting the first case of PR3ANCA associated fulminant cerebral vasculitis in an ulcerative colitis patient.

\section{Case Summary}

A 27-year-old, right-handed Caucasian male with 13-year history of UC with primary sclerosing cholangitis presented to the ER with acute onset of left sided headache, left eye proptosis, erythema, and painful eye movements without any visual changes. He reported a recent trip to Spain and denied trauma, sick contacts, urinary symptoms, fever, weight loss, sexually transmitted diseases, or similar illness in family and friends.

He was diagnosed with UC with primary sclerosing cholangitis at age of 14 following chronic diarrhea, weight loss, guaiac positive stools, and elevated GGT. The 
colonoscopy then showed pancolitis extending throughout the colon with no evidence of granuloma. Liver biopsy showed onion skinning around the bile ducts. He was initially treated with steroids, mesalamine, and ursodiol. His last flare was a year ago. He had 4-5 blood streaked loose stools per day treated with tapering dose of steroids. Since then he has had no further UC flare and has been on mesalamine and ursodiol.

Clinical examination revealed left eye ecchymosis, hemorrhagic chemosis, unilateral proptosis, with no evidence of uveitis, and normal fundus (see Figures 1(a) and 1(b)). Magnetic resonance imaging (MRI) of the face and orbit showed left eye proptosis with inflammation of the extraocular muscle and the periorbital tissue with normal brain and cavernous sinuses. He was treated with high dose steroids (one gram IV methyl prednisone for 3 days) and empiric antibiotics for orbital inflammatory syndrome and an autoimmune and infectious workup was started. His eye symptoms improved on steroids. However, on the third day of hospital admission, he complained of left arm weakness and numbness. A repeat MRI brain showed an interval development of multifocal deep gray nuclei signal abnormalities including diffusion restriction in the caudate nuclei, right globus pallidus, and posterior limb of the internal capsule (see Figure 2(a)). His CSF was essentially normal with 2 WBCS, normal protein, and glucose with few RBCs. He was empirically started on acyclovir. Within the next 36 hours, he rapidly worsened neurologically, became encephalopathic with left side hemiparesis, and had to be intubated for airway protection. His subsequent MRI brain showed further progression of lesions involving the caudate nuclei, thalami, striatum, and brainstem with new areas of hemorrhage and enhancement (Figure 2(b)). Cerebral arteriogram did not show any vasculitis.

Laboratory workup revealed mild leukocytosis with normal liver function tests, ESR, CRP, and thyroid function test. His laboratory findings were negative for HIV, hepatitis, toxoplasmosis, Saccharomyces cerevisiae, Ehrlichia, Brucella, Rocky Mountain spotted fever, Q fever, and Lyme disease. His CSF was negative for herpes simplex viruses 1 and 2, Adenovirus, Enterovirus, Cryptococcus, and Mycobacterium tuberculosis. His CSF and blood flow cytometry were negative for lymphoma/leukemia cells. Blood, stool, urine, and sputum cultures were sterile. An eye swab was negative for Chlamydia trachomatis. Skin biopsy was normal. Echocardiogram and CT scan of chest, abdomen, and pelvis were unremarkable. IgG4 was normal. Anticardiolipin antibody and beta 2 glycoprotein were both mildly elevated. He was treated empirically with antibiotics, antivirals, and steroids.

He had an ANA titer of $1: 40$ and PANCA titer of 1:320; his PR3 antibody was $157 \mathrm{AU}$ per milliliter (normal range, 0 to 19). The brain biopsy showed necrotizing vasculitis (Figure 3 ) with no evidence of granuloma. Absence of granuloma, in addition to normal lung, kidney, and sinuses ruled out GPA. Based on the serology and brain biopsy results we diagnosed PR3ANCA mediated cerebral and orbital vasculitis. He was treated similar to GPA involving the brain and eye with cyclophosphamide, plasmapheresis, and high dose of steroids. He dramatically improved with residual hemiparesis and was discharged to rehabilitation.

\section{Discussion}

The atypical PANCA seen in UC usually represents antibodies against lactoferrin, cathepsin, BPI, or elastase. PR3ANCA, antinuclear cytoplasmic antibody against proteinase 3 , the classic serological marker in granulomatosis with polyangiitis (GPA), has been noted to have an increased prevalence in ulcerative colitis. There has been a suggestion to use PR3ANCA as a serological marker for UC in the evaluation of IBD [1]. The reported incidence of PR3 ANCA in UC varies from 4 to $43 \%$ [1]. In small cohort studies PR3 ANCA has been associated with extensive colitis and shorter disease duration (average of $12 \pm 2$ years) without any gender predilection [2]. However, the incidence of small vessel vasculitis in PR3 ANCA positive UC patient cohort is not known. It is well established that in GPA PR3 antibodies with CANCA cause aggressive vasculitis which can rarely involve the eye and brain. To the best of our knowledge to date there have been no case reports of PR3ANCA mediated cerebral and orbital vasculitis in UC patients.

Cerebrovascular manifestations are seen in $0.12-4 \%$ of inflammatory bowel disease patients [17]. These include arterial and venous thrombosis, leukoencephalitis, seizures, and vasculitis. There have been only fifteen case reports of possible cerebral vasculitis in ulcerative colitis. Among these fifteen reported cases (Table 1) eleven patients had definite vasculitis based on histopathology, angiogram, or serology. Six patients had cerebral angiogram studies suggestive of vasculitis with multiple small and medium vessel narrowing, three had brain biopsy/autopsy confirming necrotizing vasculitis, one had MPO mediated systemic vasculitis with multiple brain infarcts, and one had biopsy proven skin vasculitis with multiple brain infarcts. The remaining four patients had evidence of multiple ischemic infarcts on brain imaging and vasculitis was suggested as a possible etiology based on clinical evidence. In these case series only one of the patients had MPO associated vasculitis. The limited autoimmune work up in the rest of the cases was negative. None of these patients had eye involvement. Most of these patients were treated with steroids alone or in combination with immunosuppressive therapy. Seven patients had complete recovery of neurological deficits, four patients had partial recovery, and one patient died and the diagnosis was made on the autopsy. Our patient had mild increase in antiphospholipid antibodies similar to the MPO associated vasculitis patient [6]. The elevation in these antibodies was likely a reflection of the vascular damage with vasculitis instead of the primary etiology.

The eye manifestations of UC commonly include uveitis, scleritis, and conjunctivitis which occur in about $1-6.3 \%$ of patients [18]. There are four case reports of orbital inflammatory syndrome reported in UC, of which three patients had orbital myositis and one had orbital pseudotumor [18, 19]. It is unclear if orbital vasculitis was the underlying etiology. Clinically our patient had severe orbital ecchymosis and 


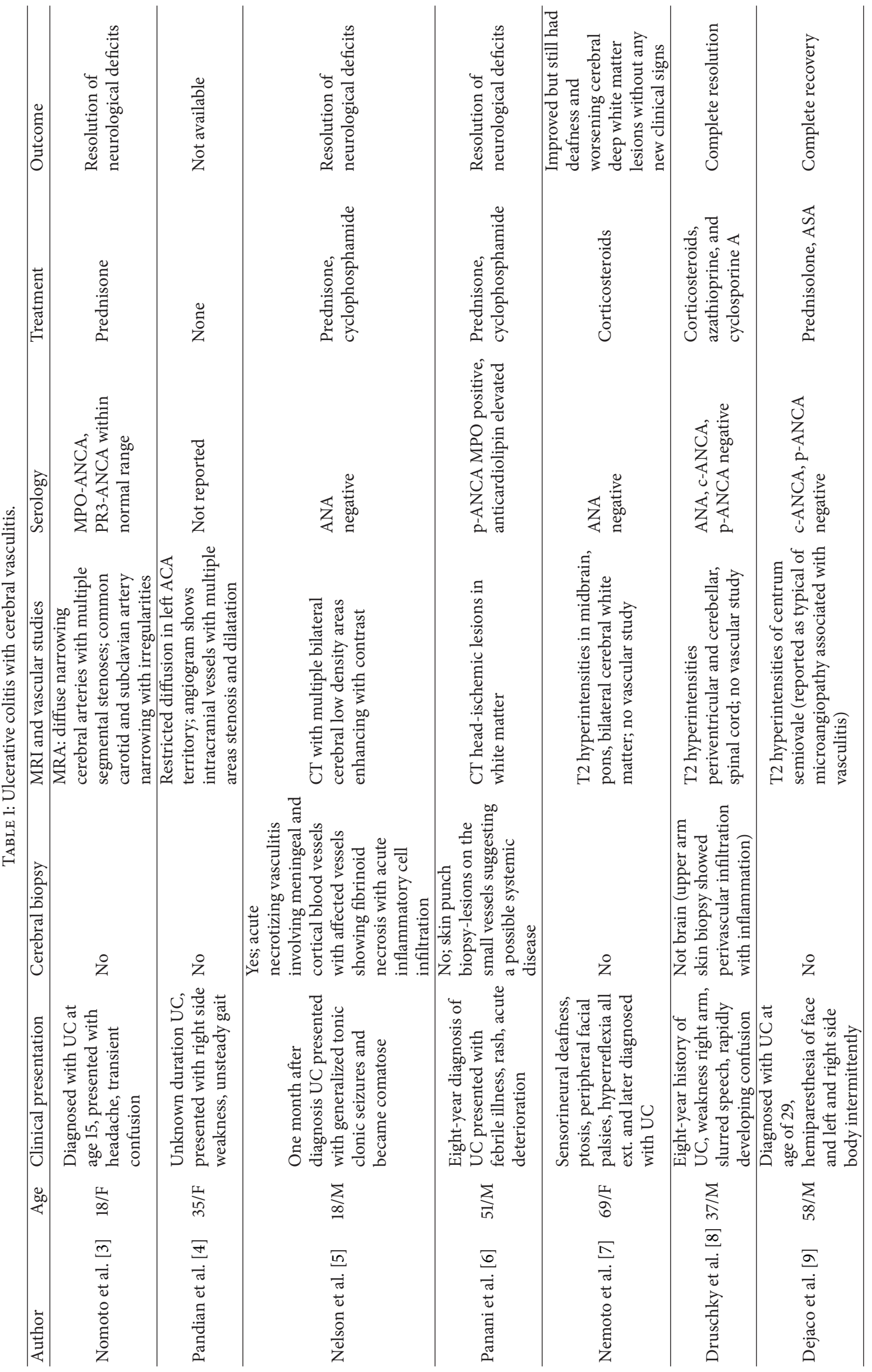




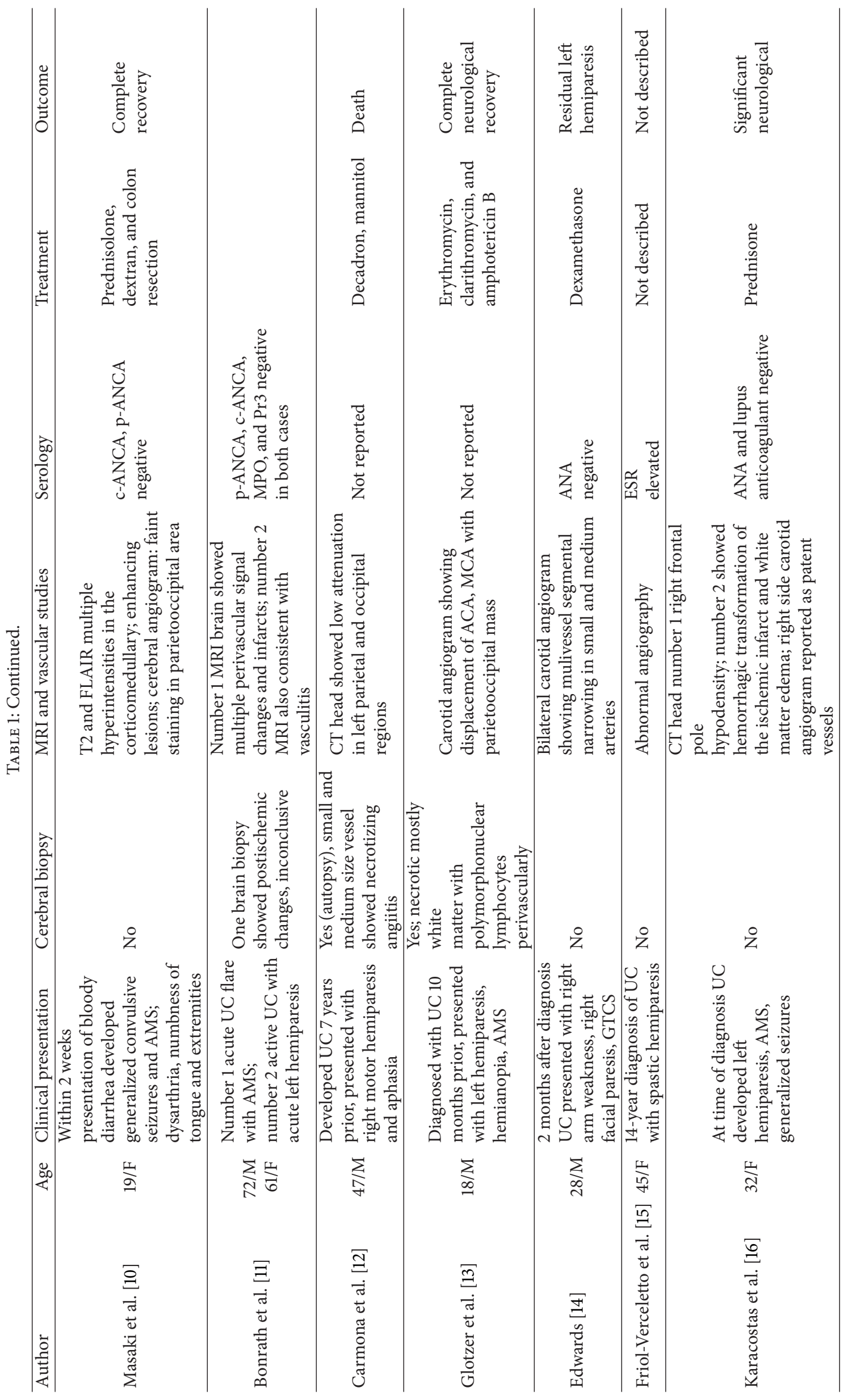




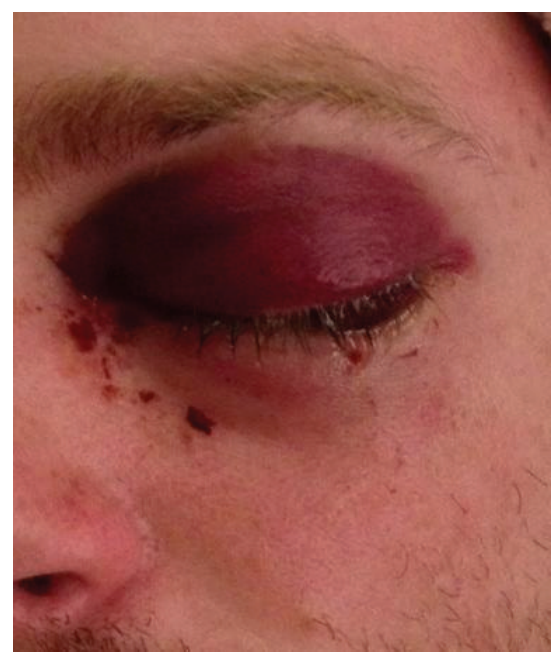

(a)

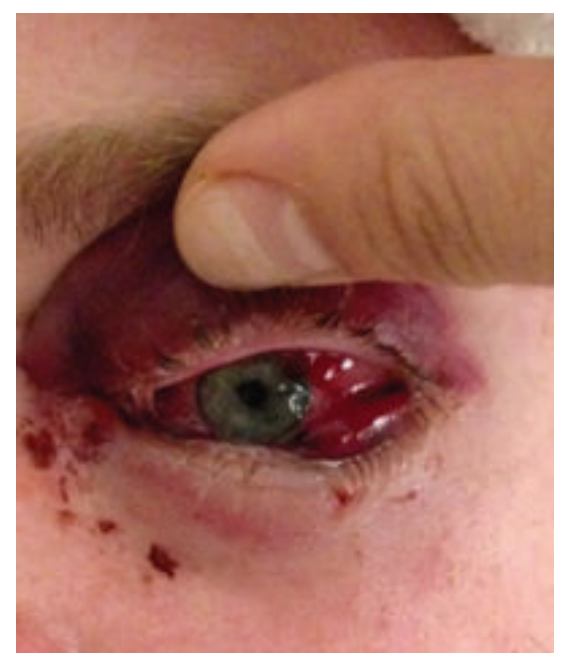

(b)

Figure 1: Day 1.

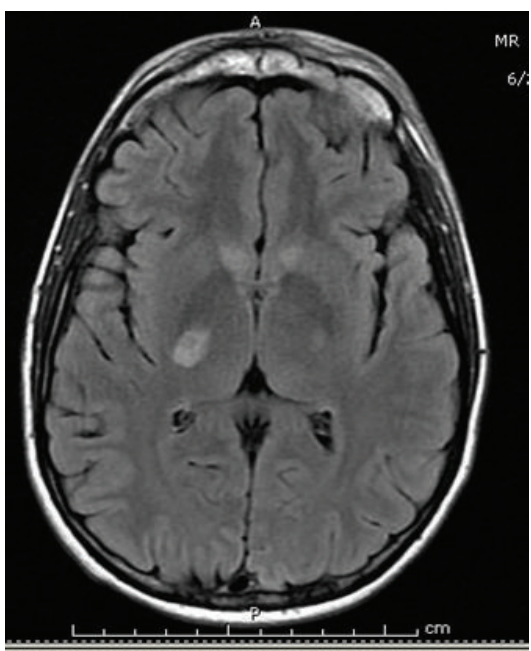

(a)

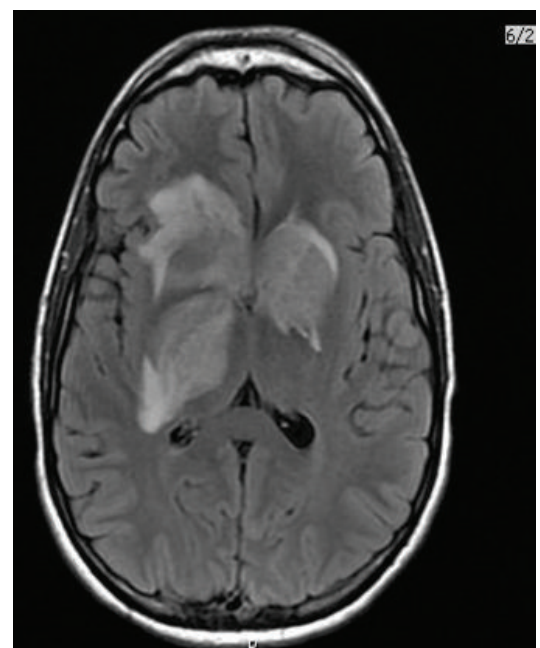

(b)

Figure 2: (a) Day 3. (b) Day 4.

conjunctival hemorrhagic chemosis which was atypical for an idiopathic orbital inflammatory syndrome. MRI showed extensive inflammatory changes in the left orbit including the periorbital soft tissue and the extraocular muscles. The brain biopsy with the positive vasculitis markers suggests the orbital inflammatory syndrome in our patient was secondary to orbital vasculitis.

Our experience in this patient suggests that PR3ANCA positivity can cause rapidly progressive cerebral vasculitis and orbital vasculitis in UC patients similar to GPA. Although there have been cases reported of ulcerative colitis later developing GPA [20] and some suggestion of an overlap between the entities there is no established relationship between GPA and UC [21]. Our patient clearly does not satisfy the diagnostic criteria to diagnose GPA.

\section{Conclusion}

Our case of cerebral vasculitis associated with ulcerative colitis is the fourth histopathologically proven case in the literature. There are no reports of orbital vasculitis and cerebral vasculitis coexisting in a patient with ulcerative colitis. This is the first case report of PR3ANCA mediated cerebral vasculitis in a UC patient presenting with orbital involvement. The fulminant course of the cerebral vasculitis in our patient highlights the need for increased awareness of the possibility of PR3ANCA associated vasculitis in UC patients to facilitate early diagnosis and treatment. We need further long term cohort studies of the subset of UC patients with PR3 ANCA to define the role of PR3 ANCA in UC and to establish any significant increase in vasculitis in this cohort. 


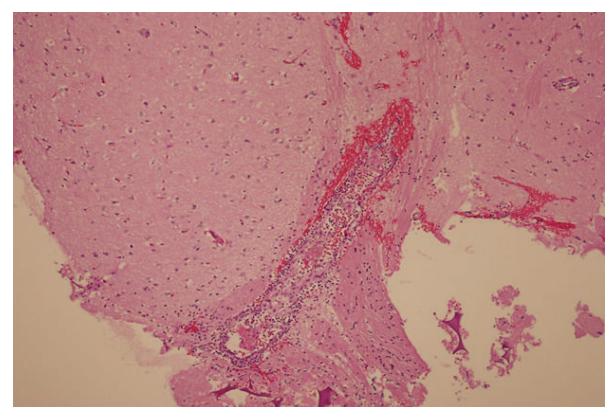

Figure 3: Brain biopsy.

We could also study if there is any increase in GPA in this patient cohort.

\section{Conflict of Interests}

The authors declare that there is no conflict of interests.

\section{References}

[1] M. Mahler, D. P. Bogdanos, P. Pavlidis et al., "PR3-ANCA: a promising biomarker for ulcerative colitis with extensive disease," Clinica Chimica Acta, vol. 424, pp. 267-273, 2013.

[2] S. Van Biervliet, C. Bonroy, S. V. Velde et al., "C-ANCA/PR-3 positive colitis in children: a distinctive form of inflammatory bowel disease or vasculitis with colitis as initial presentation?" Journal of Pediatric Gastroenterology and Nutrition, vol. 57, no. 4, pp. 489-492, 2013.

[3] T. Nomoto, T. Nagao, K. Hirabayashi et al., "Cerebral arteriopathy with extracranial artery involvement in a patient with ulcerative colitis," Journal of the Neurological Sciences, vol. 243, no. 1-2, pp. 87-89, 2006.

[4] J. D. Pandian, R. D. Henderson, J. D. O'Sullivan, and T. Rajah, "Cerebral vasculitis in ulcerative colitis," Archives of Neurology, vol. 63 , no. 5, p. 780, 2006.

[5] J. Nelson, M. M. Barron, J. E. Riggs, L. Gutmann, and S. S. Schochet Jr., "Cerebral vasculitis and ulcerative colitis," Neurology, vol. 36, no. 5, pp. 719-721, 1986.

[6] A. D. Panani, M. Grigoriadou, E. Magira, C. Roussos, and S. A. Raptis, "Perinuclear antineutrophil cytoplasmic antibody myeloperoxidase-positive vasculitis in association with ulcerative colitis," Clinical Rheumatology, vol. 25, no. 1, pp. 35-37, 2006.

[7] M. Nemoto, Y. Morita, Y. Mishima et al., "Ahl3, a third locus on mouse chromosome 17 affecting age-related hearing loss," Biochemical and Biophysical Research Communications, vol. 324, no. 4, pp. 1283-1288, 2004.

[8] A. Druschky, J. G. Heckmann, K. Druschky, W. J. Huk, F. Erbguth, and B. Neundörfer, "Severe neurological complications of ulcerative colitis," Journal of Clinical Neuroscience, vol. 9, no. 1, pp. 84-86, 2002.

[9] C. Dejaco, E. Fertl, D. Prayer et al., "Symptomatic cerebral microangiopathy preceding initial manifestation of ulcerative colitis," Digestive Diseases and Sciences, vol. 41, no. 9, pp. 18071810, 1996.

[10] T. Masaki, T. Muto, M. Shinozaki, and T. Kuroda, "Unusual cerebral complication associated with ulcerative colitis," Journal of Gastroenterology, vol. 32, no. 2, pp. 251-254, 1997.
[11] E. M. Bonrath, E. Rijcken, R. Dziewas et al., "Cerebral vasculitis as rare extraintestinal manifestation in ulcerative colitis: review of the literature and case report," Zentralblatt für Chirurgie, vol. 135, no. 4, pp. 350-353, 2010.

[12] M. A. Carmona, F. Jaume Anselmi, and J. Ramirez Rivera, "Cerebral thrombosis and vasculitis: an uncommon complication of ulcerative colitis," Boletin de la Asociacion Medica de Puerto Rico, vol. 92, no. 1-3, pp. 9-11, 2000.

[13] D. J. Glotzer, R. H. Yuan, and J. F. Patterson, "Ulcerative colitis complicated by toxic megacolon, polyserositis and hemorrhagic leukoencephalitis with recovery," Annals of Surgery, vol. 159, pp. 445-450, 1964.

[14] K. R. Edwards, "Hemorrhagic complications of cerebral arteritis," Archives of Neurology, vol. 34, no. 9, pp. 549-552, 1977.

[15] M. Friol-Vercelletto, J. M. Mussini, J. H. Bricout, and C. Magne, "Ulcero-hemorrhagic rectocolitis. Possible manifestation, angiitis of the central nervous system," Presse Medicale, vol. 13, no. 19, article 1218, 1984.

[16] D. Karacostas, J. Mavromatis, N. Artemis, and I. Milonas, "Hemorrhagic cerebral infarct and ulcerative colitis. A case report," Functional Neurology, vol. 6, no. 2, pp. 181-184, 1991.

[17] A. Lossos, Y. River, A. Eliakim, and I. Steiner, "Neurologic aspects of inflammatory bowel disease," Neurology, vol. 45, no. 3, part 1, pp. 416-421, 1995.

[18] A. Katsanos, I. Asproudis, K. H. Katsanos, A. I. Dastiridou, M. Aspiotis, and E. V. Tsianos, "Orbital and optic nerve complications of inflammatory bowel disease," Journal of Crohn's and Colitis, vol. 7, no. 9, pp. 683-693, 2013.

[19] J. Bennion, M. A. Harris, J. A. Sivak-Callcott, and J. Nguyen, "Bilateral diffuse orbital myositis in a patient with relapsing ulcerative colitis," Ophthalmic Plastic and Reconstructive Surgery, vol. 28, no. 5, pp. el19-e121, 2012.

[20] J. A. Kedziora, M. Wolff, and J. Chang, "Limited form of Wegener's granulomatosis in ulcerative colitis," American Journal of Roentgenology, vol. 125, no. 1, pp. 127-133, 1975.

[21] A. Ouazzani, Y. Sokolow, M. Hanebaly et al., "Pulmonary granulomatous necrotizing vasculitis: an extra-intestinal manifestation of ulcerative colitis or Wegener's granulomatosis?" Revue Medicale de Bruxelles, vol. 32, no. 2, pp. 93-97, 2011. 


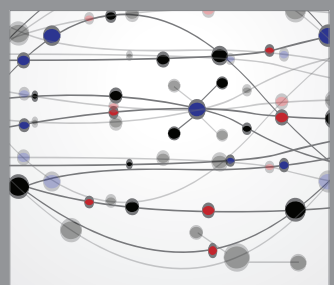

The Scientific World Journal
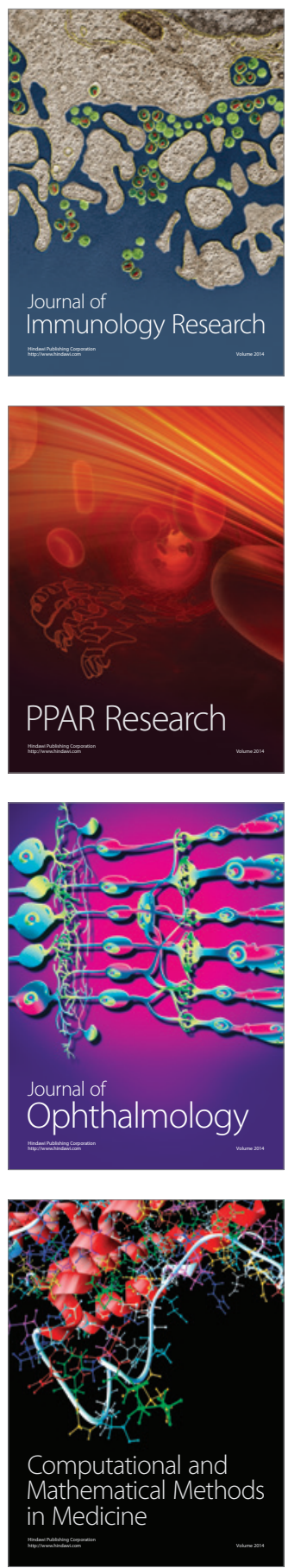

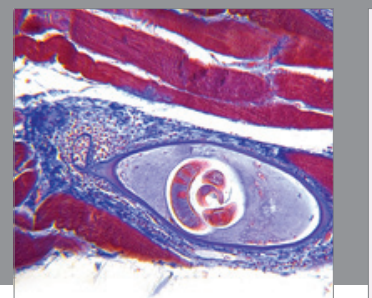

Gastroenterology

Research and Practice
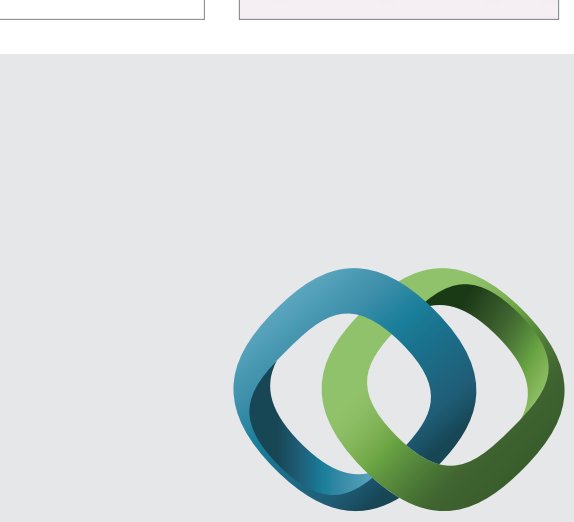

\section{Hindawi}

Submit your manuscripts at

http://www.hindawi.com
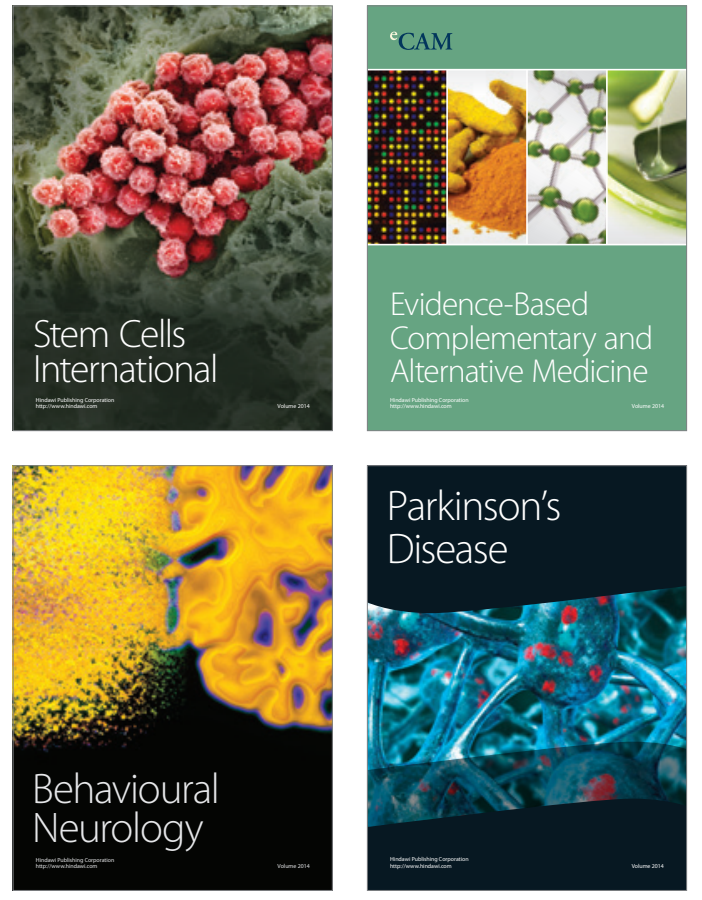
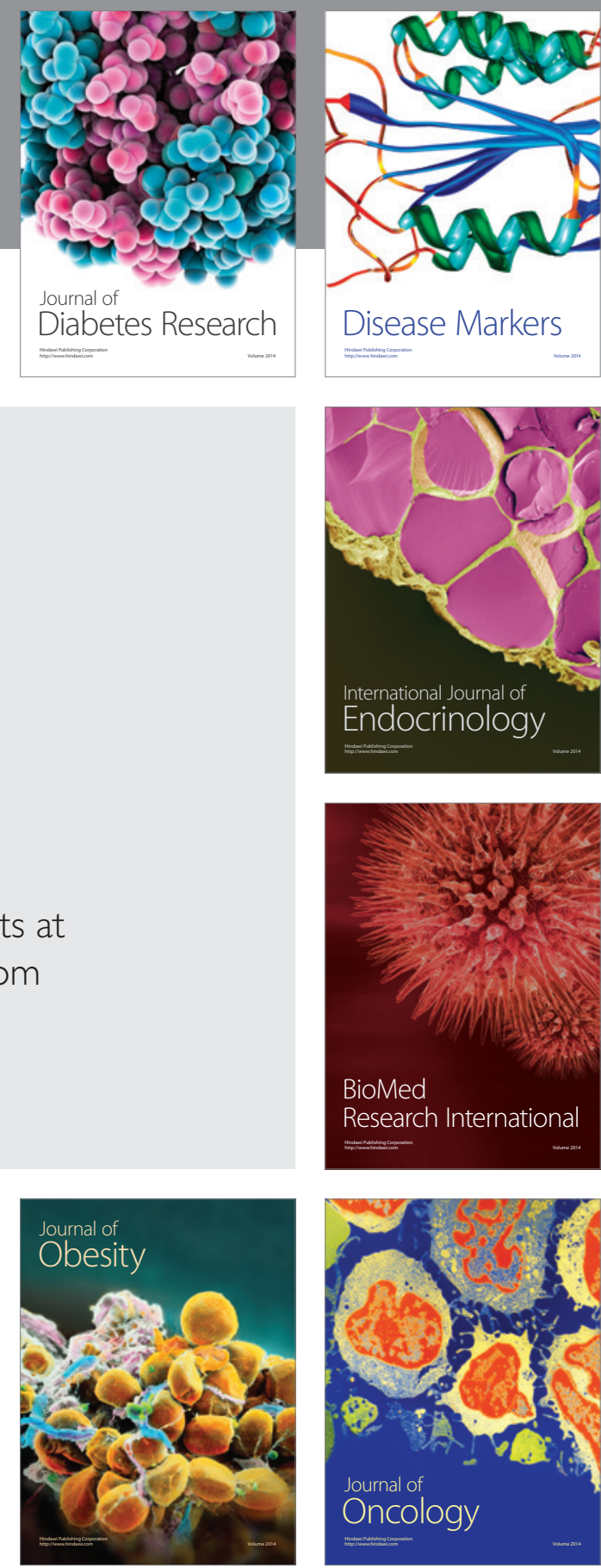

Disease Markers
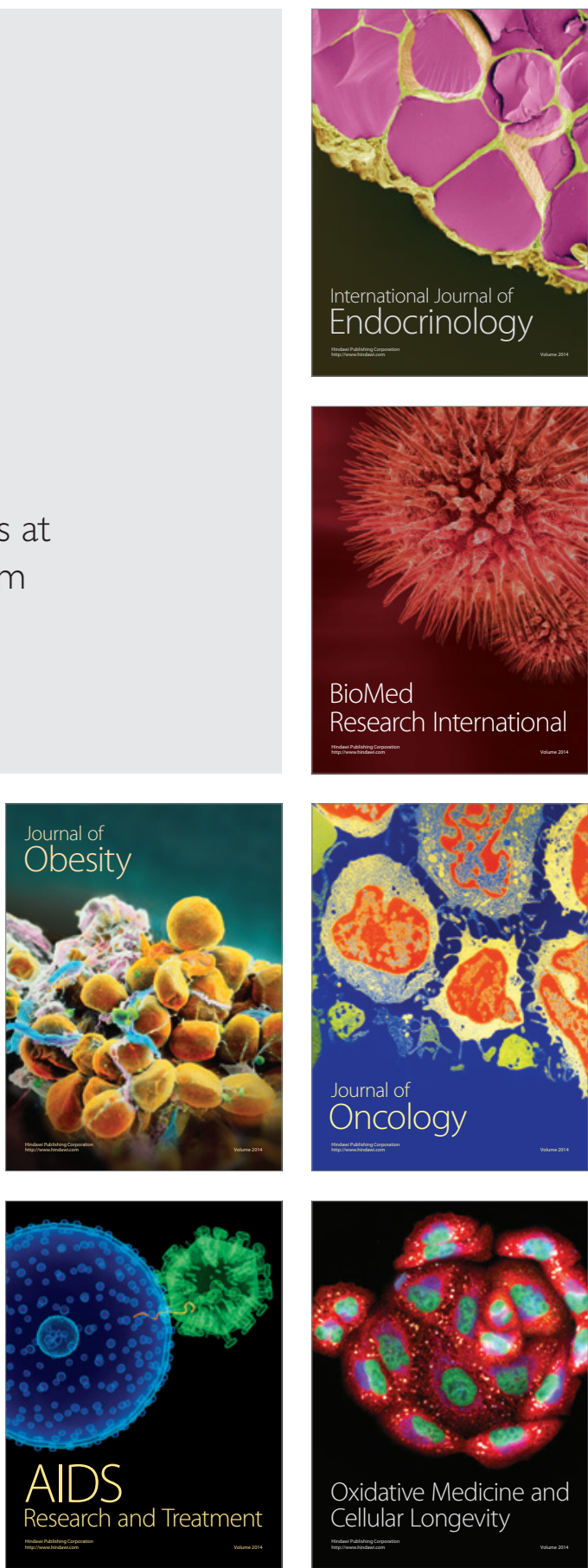